\title{
Downregulation of phosphatase of regenerating liver-3 is involved in the inhibition of proliferation and apoptosis induced by emodin in the SGC-7901 human gastric carcinoma cell line
}

\author{
ZHEN-HUA SUN and PING BU \\ Laboratory of Traditional Chinese Medicines, Medical College of Yangzhou University, \\ Yangzhou, Jiangsu 225000, P.R. China
}

Received November 7, 2011; Accepted March 7, 2012

DOI: 10.3892/etm.2012.516

\begin{abstract}
Emodin, an anthraquinone derivative isolated mainly from the root and rhizome of the medicinal plant Rheum palmatum L., was found to exert anticancer effects on various cultured cancer cells. Phosphatase of regenerating liver-3 (PRL-3), a novel gene, has been known to play an important role in the promotion of cellular proliferation as well as inhibition of apoptosis in cancer cells. However, there is relatively little information in the published literature with regard to the anticancer mechanism of emodin, and whether emodin is involved in the regulation of PRL-3 in human gastric carcinoma cells is not known. In the present study, we investigated the effects of emodin on SGC-7901 cell proliferation, apoptosis and regulation of PRL-3. The results showed that the proliferation of SGC-7901 cells was inhibited by emodin in a time- and concentration-dependent manner. The results also showed that early apoptosis rates increased in a concentration-dependent manner after emodin treatment. Furthermore, real-time quantitative PCR analysis showed that PRL-3 mRNA was significantly decreased by treatment with emodin. Western blotting showed that PRL-3 protein expression was also downregulated significantly. Overall, the present study demonstrated that emodin inhibited cell growth and induced apoptotic cell death in the SGC-7901 human gastric carcinoma cell line. Downregulation of PRL-3 is involved in the inhibition of proliferation and apoptosis induced by emodin. PRL-3 may be a new potential therapeutic target for gastric cancer using emodin.
\end{abstract}

Correspondence to: Dr Ping $\mathrm{Bu}$, Medical College of Yangzhou University, 11 Huaihai Road, Yangzhou, Jiangsu 225000, P.R. China E-mail: pingb@yahoo.cn

Key words: emodin, proliferation, apoptosis, phosphatase of regenerating liver-3, gastric cancer

\section{Introduction}

Gastric cancer is one of the most common types of cancer worldwide. Although the incidence and mortality rates of gastric cancer have decreased over the past 30 years, it remains a leading cause of cancer-related death, second only to lung cancer $(1,2)$. Infiltrative growth and metastasis are two lethal processes of gastric cancer. Despite the possibility of curative surgery, the prognosis of advanced gastric cancer remains extremely poor.

Phosphatase of regenerating liver-3 (PRL-3), also known as PTP4A3, is a novel gene that encodes a 22-kilodalton protein tyrosine phosphatase and is characteristic of a CAAX motif for prenylation at the carboxyl terminus (3). Among normal human adult tissues, PRL-3 is expressed in the heart and skeletal muscle as well as epithelial cells of the small intestine and is associated with the cell plasma membrane. Upregulation of PRL-3 is associated with the progression and eventual metastasis of several types of human cancer $(4,5)$. It is reported that high PRL-3 expression may participate in the progression and metastasis of gastric carcinoma (6). Overexpression of PRL-3 inhibits angiotensin-II-induced cell calcium mobilization and promotes cell growth. Moreover, PRL-3 activation stimulates PI3K/AKT signaling that causes resistance to stress-induced apoptosis (7). PRL-3 may be a novel molecular marker for aggressive gastric cancer (8-10).

Today, standard therapies for gastric cancer include surgery, chemotherapy and herbal therapy. These therapies are often used in combination, and they are the best hope for gastric cancer patients. At present, the use of herbal medicines for gastric cancer is widespread because of their safety and few side effects (11). Rheum palmatum L. is an ancient and well-known medicinal plant and has been used in Chinese medicine for thousands of years. Emodin (1,3,8-trihydroxy-6methylanthraquinine) is an anthraquinone derivative isolated mainly from the root and rhizome of Rheum palmatum L. Several studies have indicated that emodin exhibits a number of biological properties, including anti-proliferative (12), antiinflammatory (13), anti-pain (14) and antiviral activities (15). Emodin has also been reported to exhibit anticancer activity 
in human lung (16-18), liver (19) and ovarian cancers (20). However, there is relatively little information in the published literature with regard to the anticancer mechanism of emodin, and whether emodin is involved in the regulation of PRL-3 in human gastric carcinoma cell line SGC-7901 is not known.

In the present study, we aimed to investigate whether emodin induces the apoptosis of SGC-7901 human gastric carcinoma cells and whether PRL-3 is involved in this mechanism, thereby discovering a new potential therapeutic target for aggressive gastric cancer.

\section{Materials and methods}

Drugs and reagents. Emodin (1,3,8-trihydroxy-6-methylanthraquinine; CAS registry no. 518-82-1; purity $>98 \%$, HPLC grade) was purchased from Sangon Biotech Company, Shanghai, China. It was dissolved in 1\% DMSO (Sangon Biotech Company) and the following concentrations were prepared: $15,30,45$ and $60 \mu \mathrm{M}$. The agents were passed through a $0.22-\mu \mathrm{m}$ filter for sterilization. The molecular formula of emodin is $\mathrm{C}_{15} \mathrm{H}_{10} \mathrm{O}_{5}$ and its molecular weight is 270.24 .

RPMI-1640 medium was purchased from Gibco Company, USA. Trypsin, DMSO, RIPA, PMSF and the ECL Staining kit were purchased from Sangon Biotech Company. MTT (methyl thiazolyl tetrazolium) was purchased from Sigma-Aldrich Company, USA, and penicillin $\mathrm{G}$ and streptomycin sulfate were purchased from Lukang Phamaceutical Company, Shandong, China. TRIzol ${ }^{\circledR}$ reagent was purchased from Invitrogen Company (Carlsbad, CA, USA), and the SYBR ${ }^{\circledR}$ PrimeScript RT-PCR kit II was purchased from Takara Biotechnology Company, Dalian, China. The BCA protein detection kit and goat anti-mouse IgG-HRP second antibody were purchased from Boster Bio-Engineering Company, Wuhan, China. The Annexin V-FITC apoptosis detection kit was purchased from KeyGEN Bio-Technology Company, Nanjing, China. Antibodies for $\beta$-actin and PRL-3 were purchased from Santa Cruz Biotechnology, Inc. (Santa Cruz, CA, USA).

Cell culture and treatment. The SGC-7901 human gastric carcinoma cell line was obtained from the Institute of Biochemistry and Cell Biology (Shanghai Institutes for Biological Sciences, China). Cells were cultured in RPMI-1640 supplemented with $10 \%$ heat-inactivated fetal bovine serum, $100 \mathrm{U} / \mathrm{ml}$ penicillin $\mathrm{G}$ and $100 \mu \mathrm{g} / \mathrm{ml}$ streptomycin sulfate. The cell line was supplied with fresh medium every 1-2 days and grown at $37^{\circ} \mathrm{C}$ in a humidified atmosphere containing $5 \% \mathrm{CO}_{2}$. After preincubation in RPMI-1640 medium for $24 \mathrm{~h}$, cells were divided into five groups: A group (control group, no-treatment); B group (emodin treatment group, $15 \mu \mathrm{M}$ ); $\mathrm{C}$ group (emodin treatment group, $30 \mu \mathrm{M}$ ); D group (emodin treatment group, $45 \mu \mathrm{M}$ ) and $\mathrm{E}$ group (emodin treatment group, $60 \mu \mathrm{M}$ ). Prior to treatment, the cells were grown to $80-90 \%$ confluency.

MTT assay for cell viability. Cell viability was determined by MTT assay. Briefly, the cells $\left(6 \times 10^{3}\right)$ were seeded in 96-well plates and were incubated at $37^{\circ} \mathrm{C}$ in $5 \% \mathrm{CO}_{2} / 95 \%$ air. After $24 \mathrm{~h}$, these cells were treated with emodin at different concentrations $(15,30,45$ and $60 \mu \mathrm{M})$ and incubated for 24,48 and $72 \mathrm{~h}$. The control group was treated with the equivalent amount of dimethyl sulphoxide (DMSO; the maximum of $0.2 \%$ of the

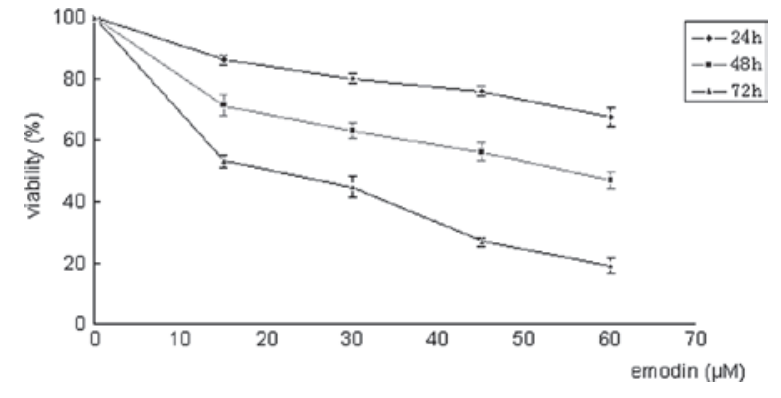

Figure 1. Effect of emodin on the cell viability of SGC-7901 cells. Cells were incubated with different concentrations of emodin $(15,30,45$ and $60 \mu \mathrm{M})$ for 24,48 and 72 h. Cell viability was evaluated with MTT assay. Concentrationdependent and time-dependent inhibition of SGC-7901 cell growth was observed $(\mathrm{P}<0.01$ compared to control).

assay mixture was used). Then, $20 \mu 1$ MTT solution $(5 \mathrm{mg} / \mathrm{ml})$ was added to each well and incubation was carried out at $37^{\circ} \mathrm{C}$ for $4 \mathrm{~h}$. The medium was removed, formazan was dissolved in DMSO and the absorbance was detected at $570 \mathrm{~nm}$ using a multiscan reader (Labsystems, Helsinki, Finland). Survival ratio (\%) was calculated using the following equation:

$$
\text { Survival ratio }(\%)=\left(\mathrm{A}_{\text {Treatment }} / \mathrm{A}_{\text {Control }}\right) \times 100 \% \text {. }
$$

Apoptotic cell determination by Annexin V/PI staining assay. Cell apoptosis induced by emodin was detected using Annexin V-FITC Apoptosis Detection kit. A total of $1 \times 10^{4}$ SGC-7901 cells/well were seeded into 6-well plates for $24 \mathrm{~h}$ of incubation at $37^{\circ} \mathrm{C}$. Various concentrations of emodin were added to the wells and incubation was carried out for an additional $24 \mathrm{~h}$ (the final concentrations of emodin were 15 , $30,45$ and $60 \mu \mathrm{M})$. The control group was treated with the equivalent amount of DMSO (the maximum of $0.2 \%$ of the assay mixture was used). Following trypsinization and gentle washing of the cells once with medium, the cells were washed with PBS twice, and then resuspended in $200 \mu$ l of binding buffer. Annexin V-FITC $(10 \mu \mathrm{l})$ and propidium iodide (PI) $(5 \mu \mathrm{l})$ were added to the resuspended cells. After incubation at room temperature for $15 \mathrm{~min}$ in the dark, $300 \mu \mathrm{l}$ of binding buffer was added to the resuspended cells, and then the stained cells were analyzed by flow cytometry.

Analysis of PRL-3 mRNA by real-time quantitative PCR. After treatment with 15, 30, 45 and $60 \mu \mathrm{M}$ emodin for $24 \mathrm{~h}$, SGC-7901 cells were harvested by trypsinization and RNA was extracted using TRIzol ${ }^{\circledR}$ reagent. Reverse transcription of $0.5 \mu \mathrm{g}$ RNA was carried out according to $\mathrm{SYBR}^{\circledR}$ PrimeScript RT-PCR kit II. Real-time fluorescence detection was carried out with Applied Biosystems 7500 Real Time PCR System (Applied Biosystems, Foster City, CA, USA). The specific primers were 5'-CACGCTCAGCACCTTCATTG-3' (forward) and 5'-TTGTCATAGGTCACTTCACACACAC-3' (reverse) for PRL-3 and 5'-GCACCGTCAAGGCTGAGAAC-3' (forward) and 5'-TGGTGAAGACGCCAGTGGA-3' (reverse) for GAPDH. The reaction volume was $20 \mu 1$, and $100 \mathrm{ng}$ cDNA was used as template. The amplification parameters were the following: optimization steps at $95^{\circ} \mathrm{C}$ for $30 \mathrm{sec}$, followed by 40 cycles of $95^{\circ} \mathrm{C}$ for $5 \mathrm{sec}$ (denaturation) and $64^{\circ} \mathrm{C}$ for $34 \mathrm{sec}$ 
A
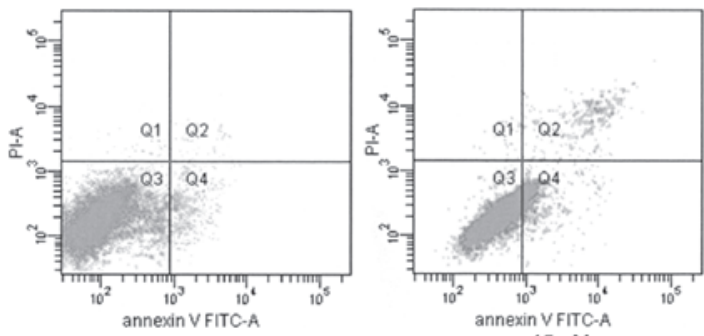

control
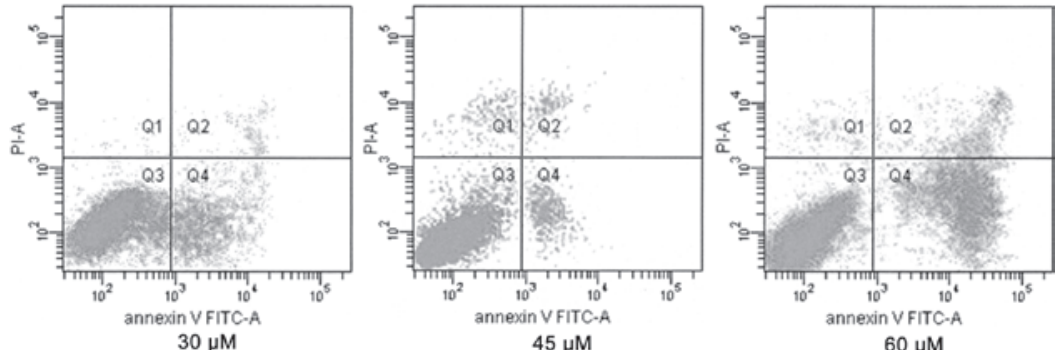

B

$45 \mu \mathrm{M}$

$60 \mu \mathrm{M}$

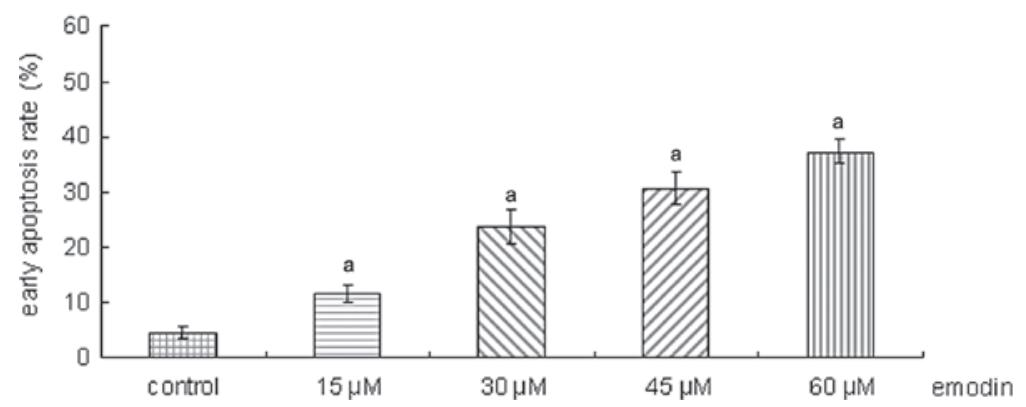

Figure 2. Apoptosis induced by emodin in SGC-7901 cells. (A) SGC-7901 cells were treated with 15, 30, 45 and $60 \mu \mathrm{M}$ emodin for $24 \mathrm{~h}$. Cells were then collected and stained with FITC-Annexin V/PI to detect cell death phenomena. The y-axis indicates PI-labeled population and the x-axis indicates FITC-labeled Annexin V-positive cells. Cells in the lower right area indicate apoptotic cells. One representative experiment out of three is shown. (B) Apoptotic rates of SGC-7901 cells induced by emodin. ${ }^{\mathrm{a}} \mathrm{P}<0.01$ compared to the control.

(annealing and extension). Amplifications were normalized to GAPDH of the respective treatment. The relative mRNA expression level of the PRL-3 gene was determined by the $2^{-\Delta C}$ x 100\% method, where $\Delta \mathrm{Ct}=\mathrm{Ct}(\mathrm{PRL}-3)-\mathrm{Ct}(\mathrm{GAPDH})(2)$.

Detection of the expression of PRL-3 by western blot analysis. After treatment with 15, 30, 45 and $60 \mu \mathrm{M}$ emodin for $24 \mathrm{~h}$, the cells were washed twice using ice-cold PBS ( $\mathrm{pH}$ 7.4). The cells were lysed in RIPA buffer $(150 \mathrm{mmol} / \mathrm{l} \mathrm{NaCl}, 50 \mathrm{M}$ Tris, $1 \mathrm{mmol} / 1$ EDTA, $1 \%$ NP-40, 0.5\% sodium deoxycholate and $0.1 \%$ SDS, $\mathrm{pH}$ 7.4) containing protease inhibitor cocktail. Cell lysates were then clarified by centrifugation at $12,000 \mathrm{x} \mathrm{g}$ for $10 \mathrm{~min}$ at $4^{\circ} \mathrm{C}$. Protein concentrations were determined using the BCA method. Equal amounts of denatured protein were loaded; proteins were electrophoresed on $12 \%$ SDS-polyacrylamide gels and transferred to a PVDF membrane. After being blocked with $5 \%$ fat-free dry milk in Tris-buffered saline (TBS) for $3 \mathrm{~h}$ at room temperature, primary antibodies that detect PRL-3 and $\beta$-actin were incubated with membranes overnight at $4^{\circ} \mathrm{C}$. The membranes were then incubated with horseradish peroxidase (HRP)-conjugated secondary antibody for $1 \mathrm{~h}$ at room temperature, and then immunoreactive bands were detected using the ECL method.
Image processing was performed using the software Quantity One. Values were corrected in accordance with the absorbency of the internal control ( $\beta$-actin).

Statistical analysis. All results were expressed as the means \pm SEM of at least three independent experiments and were analyzed using SPSS 10.0 software. Statistical significance was assessed by ANOVA. Effects were analyzed in more detail with least significant difference (LSD) post hoc tests. P-values $<0.05$ denoted statistical significance.

\section{Results}

Emodin inhibits SGC-7901 cell viability in a concentrationand time-dependent manner. To investigate the inhibitory effect of emodin on SGC-7901 cells, MTT assay was used to quantify the effect of emodin on SGC-7901 cell growth. As shown in Fig. 1, emodin caused a decrease in cell viability in the SGC-7901 cells in a concentration- and time-dependent manner when compared to the control.

Effects of emodin on early apoptosis by Annexin VIPI staining. Early apoptotic changes were identified using Annexin V/PI 


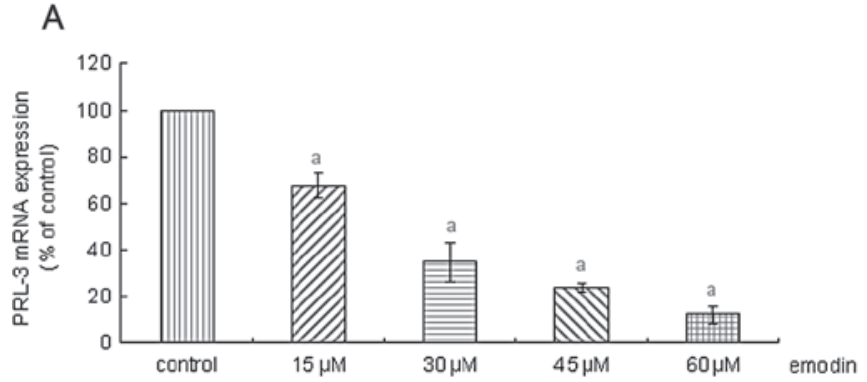

B

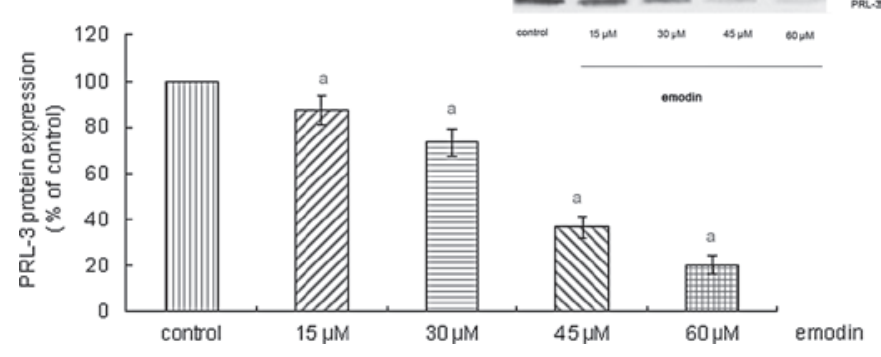

Figure 3. Effects of emodin on the expression of PRL-3. (A) Inhibitory effects of emodin on the mRNA expression of PRL-3 in SGC-7901 cells measured by real-time quantitative PCR. The fold change of PRL-3 expression is calculated by the $2^{-\Delta C t}$ method, and expressed as mean fold induction over the control group that has been normalized to $100 \%$. (B) Western blot analysis showed that emodin downregulated PRL-3 expression in a concentrationdependent (15-60 $\mu \mathrm{M})$ manner. Blots are representative of three independently performed experiments. $\beta$-actin expression was also analyzed as a control for protein loading. ${ }^{a}$ Significantly different when compared to control $(\mathrm{P}<0.01)$.

staining. As shown in Fig. 2, after treated with 15, 30, 45 and $60 \mu \mathrm{M}$ emodin for $24 \mathrm{~h}$, respectively, the percentage of early apoptotic cells was increased (4.50 to $11.63,23.58,30.45$ and $37.25 \%$, respectively). These results suggest that the induction of apoptosis was involved in the antitumor effect of emodin.

Effects of emodin on PRL-3 mRNA expression in SGC-7901 cells. Emodin-treated SGC-7901 cells showed a significant decrease in PRL-3 mRNA expression when compared to the control $(\mathrm{P}<0.01)$. As shown in Fig. 3A, emodin at the concentration of $15 \mu \mathrm{M}$ decreased PRL-3 mRNA of SGC-7901 cells by $32.47 \%$, while at a concentration of $60 \mu \mathrm{M}$, the levels of PRL-3 mRNA were decreased by $87.72 \%$.

Effects of emodin on PRL-3 protein expression in SGC-7901 cells. As shown in Fig. 3B, after SGC-7901 cells were treated with 15, 30, 45 and $60 \mu \mathrm{M}$ emodin for $24 \mathrm{~h}$, respectively, PRL-3 expression was downregulated in a concentration-dependent manner $(15-60 \mu \mathrm{M})$. The emodin-mediated changes in the protein levels of PRL-3 coincided well with the mRNA levels as evidenced by the real-time quantitative PCR results.

\section{Discussion}

Emodin, 1,3,8-trihydroxy-6-methylanthraquinine, is an anthraquinone derivative isolated mainly from the root and rhizome of the medicinal plant Rheum palmatum L. Emodin exhibits various biological activities, such as anti-proliferative, anti-inflammatory, antibacterial, cell cycle arrest, immunosup- pressive, antiviral and anticancer activity $(21,22)$, which have been well documented in several cell lines in recent years, including K562 human chronic myelocytic leukemia cells (23), human breast carcinoma cells (24) and hepatoma cells (19). Phosphatase of regenerating liver-3 (PRL-3), also known as PTP4A3, encodes a $22-\mathrm{kDa}$ protein tyrosine phosphatase and is characteristic of a CAAX motif for prenylation at the carboxyl terminus (25). The PRLs are members of the PTP superfamily (26) and have an N-terminal catalytic domain containing the signature PTP active site sequence CX5R. The overexpression of PRL-3 has been frequently observed in a variety of cancers, as well as in metastatic cancers (27-29). PRL-3 levels were further confirmed to be correlated with cancer progression and pathological stage (30). However, there is relatively little information in the published literature with regard to the relationship between emodin and PRL-3. The effect of emodin on the apoptosis and changes in PRL-3 expression in the SGC-7901 human gastric carcinoma cell line has yet to be studied.

In the present study, we used the SGC-7901 human gastric carcinoma cell line to study the anticancer effect of emodin, and demonstrated that emodin exhibits an anticancer effect against gastric cancer. Emodin at concentrations of 15, 30, 45 and $60 \mu \mathrm{M}$ was used. Cell viability was inhibited by $13.71 \%$ following treatment with the lower concentration $(15 \mu \mathrm{M})$. Maximum inhibition (80.98\%) was observed with treatment of $60 \mu \mathrm{M}$. Different concentrations of emodin significantly inhibited the proliferation of SGC-7901 cells concentration- and time-dependently, compared to the control group $(\mathrm{P}<0.01)$.

In addition to inhibiting tumor cell growth, emodin also showed good ability in inducing cell apoptosis. Apoptosis is widely known as programmed cell death and is essential for normal development and for the maintenance of homeostasis. Accumulated evidence shows that many Chinese herbs have antitumor properties and induction of apoptosis is one of the mechanisms. To further confirm the apoptosis induced by emodin, Annexin V/PI staining assay was used. In our study, after treatment with $15,30,45$ and $60 \mu \mathrm{M}$ of emodin for $24 \mathrm{~h}$, respectively, the percentage of apoptotic cells was increased (4.50 to $11.63,23.58,30.45$ and $37.25 \%$, respectively) and the percentage of necrotic cells (Fig. 2A, right upper section of the fluorocytogram) was increased slightly. These results suggest that emodin displays anticancer activity in SGC-7901 gastric carcinoma cells and that apoptosis induction by emodin was involved in its antitumor effect.

To further investigate the effectiveness of emodin on the transcriptional levels of PRL-3, which has been known to play an important role in the promotion of cellular proliferation as well as inhibition of apoptosis in cancer cells, we also applied real-time quantitative PCR to cells treated with emodin. The main advantage of real-time quantitative PCR over conventional PCR is that with real-time quantitative PCR the starting template copy number is determined with accuracy and high sensitivity over a wide dynamic range. In the present study, quantification of PRL-3 mRNA demonstrated that emodin concentration-dependently downregulated the expression of PRL-3 mRNA in SGC-7901 cells, indicating that emodin may regulate PRL-3 expression at the transcriptional level. 
It is well known that regulation at the mRNA level does not always predict regulation at the protein level. As such, we investigated the effects of emodin on the expression levels of PRL-3 protein by western blotting. The results showed that after treatment with $15,30,45$ and $60 \mu \mathrm{M}$ of emodin for $24 \mathrm{~h}$, respectively, the expression of PRL-3 decreased significantly. Results showed that the downregulation of PRL-3 after emodin treatment was in a concentration-dependent manner. The emodin-mediated changes in the protein levels of PRL-3 coincided well with the mRNA levels as evidenced by the real-time quantitative PCR results. The downregulation of PRL-3 protein was involved in the emodin-induced apoptosis of SGC-7901 cells.

In conclusion, the present study demonstrated that emodin inhibited cell growth and induced apoptotic cell death in the SGC-7901 human gastric cancer cell line. PRL-3 expression levels decreased when SCG-7901 cells were treated with emodin, which was positively related to the apoptotic rate. These findings indicated that downregulation of PRL-3 is involved in the inhibition of proliferation and apoptosis induced by emodin in the SGC-7901 human gastric carcinoma cell line. PRL-3 may be a novel molecular marker for aggressive gastric cancer.

\section{Acknowledgements}

This study was supported by the Medical College of Yangzhou University, China. The authors thank Dr Chen Hong for his useful suggestions and excellent technical assistance.

\section{References}

1. Yamashita K, Sakuramoto S and Watanabe M: Genomic and epigenetic profiles of gastric cancer: potential diagnostic and therapeutic applications. Surg Today 41: 24-38, 2011.

2. Stams WA, den Boer ML, Beverloo HB, et al: Sensitivity to $\mathrm{L}$-asparaginase is not associated with expression levels of asparagine synthetase in $\mathrm{t}(12 ; 21)^{+}$pediatric ALL. Blood 101: 2743-2747, 2003.

3. Al-Aidaroos AQ and Zeng Q: PRL-3 phosphatase and cancer metastasis. J Cell Biochem 111: 1087-1098, 2010.

4. Guzinska-Ustymowicz K and Pryczynicz A: PRL-3, an emerging marker of carcinogenesis, is strongly associated with poor prognosis. Anticancer Agents Med Chem 11: 99-108, 2011.

5. Xu Y, Zhu M, Zhang S, Liu H, Li T and Qin C: Expression and prognostic value of PRL-3 in human intrahepatic cholangiocarcinoma. Pathol Oncol Res 16: 169-175, 2010.

6. Dai N, Lu AP, Shou CC and Li JY: Expression of phosphatase regenerating liver 3 is an independent prognostic indicator for gastric cancer. World J Gastroenterol 15: 1499-1505, 2009.

7. Bishop JD, Nien WL, Dauphinee SM and Too CK: Prolactin activates mammalian target-of-rapamycin through phosphatidylinositol 3-kinase and stimulates phosphorylation of p70S6K and 4E-binding protein-1 in lymphoma cells. J Endocrinol 190: 307-312, 2006

8. Cai SR, Wang Z, Chen CQ, et al: Role of silencing phosphatase of regenerationg liver-3 expression by microRNA interference in the growth of gastric cancer. Chin Med J (Engl) 121: 2534-2538, 2008.

9. Wang H, Vardy LA, Tan CP, et al: PCBP1 suppresses the translation of metastasis-associated PRL-3 phosphatase. Cancer Cell 18 $52-62,2010$.
10. Wang Z, Cai SR, He YL, et al: High expression of PRL-3 can promote growth of gastric cancer and exhibits a poor prognostic impact on patients. Ann Surg Oncol 16: 208-219, 2009.

11. Olaku $\mathrm{O}$ and White JD: Herbal therapy use by cancer patients: a literature review on case reports. Eur J Cancer 47: 508-514, 2011.

12. Wang QJ, Cai XB, Liu MH, Hu H, Tan XJ and Jing XB: Apoptosis induced by emodin is associated with alterations of intracellular acidification and reactive oxygen species in EC-109 cells. Biochem Cell Biol 88: 767-774, 2010.

13. Meng G, Liu Y, Lou C and Yang H: Emodin suppresses lipopolysaccharide-induced pro-inflammatory responses and NF-kappaB activation by disrupting lipid rafts in CD14-negative endothelial cells. Br J Pharmacol 161: 1628-1644, 2010.

14. Gao Y, Liu H, Deng L, et al: Effect of emodin on neuropathic pain transmission mediated by $\mathrm{P} 2 \mathrm{X} 2 / 3$ receptor of primary sensory neurons. Brain Res Bull 84: 406-413, 2011.

15. Xiong HR, Luo J, Hou W, Xiao H and Yang ZQ: The effect of emodin, an anthraquinone derivative extracted from the roots of Rheum tanguticum, against herpes simplex virus in vitro and in vivo. J Ethnopharmacol 133: 718-723, 2011.

16. Ko JC, Su YJ, Lin ST, et al: Emodin enhances cisplatin-induced cytotoxicity via down-regulation of ERCC1 and inactivation of ERK1/2. Lung Cancer 69: 155-164, 2010.

17. Lai JM, Chang JT, Wen CL and Hsu SL: Emodin induces a reactive oxygen species-dependent and ATM-p53-Bax mediated cytotoxicity in lung cancer cells. Eur J Pharmacol 623: 1-9, 2009.

18. Su YJ, Tsai MS, Kuo YH, et al: Role of Rad51 down-regulation and extracellular signal-regulated kinases 1 and 2 inactivation in emodin and mitomycin C-induced synergistic cytotoxicity in human non-small-cell lung cancer cells. Mol Pharmacol 77: 633-643, 2010

19. Hsu CM, Hsu YA, Tsai Y, et al: Emodin inhibits the growth of hepatoma cells: finding the common anti-cancer pathway using Huh7, Hep3B, and HepG2 cells. Biochem Biophys Res Commun 392: 473-478, 2010.

20. Li J, Liu P, Mao H, Wanga A and Zhang X: Emodin sensitizes paclitaxel-resistant human ovarian cancer cells to paclitaxelinduced apoptosis in vitro. Oncol Rep 21: 1605-1610, 2009.

21. Lin SY, Lai WW, Ho CC, et al: Emodin induces apoptosis of human tongue squamous cancer SCC-4 cells through reactive oxygen species and mitochondria-dependent pathways. Anticancer Res 29: 327-335, 2009.

22. Wang W, Sun YP, Huang XZ, et al: Emodin enhances sensitivity of gallbladder cancer cells to platinum drugs via glutathion depletion and MRP1 downregulation. Biochem Pharmacol 79: 1134-1140, 2010.

23. Chun-Guang W, Jun-Qing Y, Bei-Zhong L, et al: Anti-tumor activity of emodin against human chronic myelocytic leukemia K562 cell lines in vitro and in vivo. Eur J Pharmacol 627: 33-41, 2010.

24. Huang Z, Chen G and Shi P: Effects of emodin on the gene expression profiling of human breast carcinoma cells. Cancer Detect Prev 32: 286-291, 2009.

25. Pascaru M, Tanase C, Vacaru AM, et al: Analysis of molecular determinants of PRL-3. J Cell Mol Med 13: 3141-3150, 2009.

26. Bessette DC, Qiu D and Pallen CJ: PRL PTPs: mediators and markers of cancer progression. Cancer Metastasis Rev 27: 231-252, 2008

27. Hao RT, Zhang XH, Pan YF, et al: Prognostic and metastatic value of phosphatase of regenerating liver-3 in invasive breast cancer. J Cancer Res Clin Oncol 136: 1349-1357, 2010.

28. Reich R, Hadar S and Davidson B: Expression and clinical role of protein of regenerating liver (PRL) phosphatases in ovarian carcinoma. Int J Mol Sci 12: 1133-1145, 2011.

29. Ren T, Jiang B, Xing X, et al: Prognostic significance of phosphatase of regenerating liver-3 expression in ovarian cancer. Pathol Oncol Res 15: 555-560, 2009.

30. Ooki A, Yamashita K, Kikuchi S, Sakuramoto S, Katada N and Watanabe M: Phosphatase of regenerating liver-3 as a prognostic biomarker in histologically node-negative gastric cancer. Oncol Rep 21: 1467-1475, 2009. 